\title{
Temperature-Dependence of Debye-Waller Factors of Semiconductors Presented in Terms of Cumulant Expansion
}

\author{
Nguyen Cong Toan and Nguyen Van Hung* \\ Department of Physics, VNU University of Science, Vietnam National University, Vietnam
}

*Corresponding author: Nguyen Van Hung, Department of Physics, VNU University of Science, Vietnam National University, Hanoi, 334 Nguyen Trai, Thanh Xuan, Hanoi, Vietnam.
Received Date: November 05, 2019

Published Date: November 13, 2019

\begin{abstract}
This work studies temperature-dependence of Debye-Waller factors of semiconductors presented in terms of cumulant expansion in extended X-ray absorption fine structure (EXAFS). The advances in these studies are succeeded based on further development of the anharmonic correlated Einstein model derived primary for fcc crystals into the one for applying to semiconductors and for creating a method having the advantage that all considered quantities are provided based on only the calculation or measurement of second cumulants. Analytical expressions of three first EXAFS cumulants have been derived. They describe the thermodynamic and anharmonic properties and contribute to accurate structural determination of semiconductors. The theory simplifies the many-body task into the one of one-dimensional model with taking the many-body effects into account based on the first shell near neighbor contributions to the vibration between absorber and backscattered atoms. Morse potential is assumed to describe single-pair atomic interaction. The results calculated using the present theory and those obtained by the created method for Ge having diamond structure are found to be in good agreement with experiment and with those of other theorie.
\end{abstract}

Keywords: Temperature dependence; Debye-Waller factor; Cumulant expansion; Anharmonic correlated einstein model; Semiconductor

\section{Introduction}

Extended X-ray Absorption Fine Structure (EXAFS) has developed into a powerful technique for providing information on the local atomic structure and thermal effects of substances. The formalism for including anharmonic effects in EXAFS is often based on the cumulant expansion approach [1] from which the expression for anharmonic EXAFS is given by

$$
\chi(k)=F(k) \frac{e^{-2 R / \lambda(k)}}{k R^{2}} \operatorname{Im}\left\{e^{i \Phi(k)} \exp \left[2 i k R+\frac{(2 i k)^{n}}{n !} \sigma^{(n)}\right]\right\}
$$

where $\mathrm{F}(\mathrm{k})$ is the real atomic backscattering amplitude, $\mathrm{k}$ and $\lambda$ are the wave number and mean free path of the photoelectron, respectively, $\phi$ is net phase shift and $\sigma^{(\mathrm{n})}(\mathrm{n}=1,2,3, \ldots)$ are the cumulants describing Debye-Waller factor (DWF).

In this expression of EXAFS the even cumulants contribute to the amplitude, the odd ones to the phase of EXAFS spectra, and they also account for the net thermal expansion (first cumulant), the mean square relative displacement (MSRD) (second cumulant) and the asymmetry of the pair distribution function (third cumulant).
Consequently, the lack of the precise DWFs has been one of the biggest limitations not only to accurate structural determinations but also to specifications of the thermodynamic and anharmonic properties of materials from EXAFS data. Therefore, investigation of DWFs is of great interest.

The purpose of this work is to study temperature-dependence of DWFs of semiconductors presented in terms of cumulant expansion in EXAFS. The most advantageous developments in these studies are presented in Section 2. They are succeeded based on the further development of the anharmonic correlated Einstein model (ACEM) [2] derived primary for fcc crystals, into the one for applying to semiconductors and for creating a method having the advantage that all considered quantities are provided based on only the calculation or measurement of second cumulants. Analytical expressions of three first EXAFS cumulants have been derived. They describe the thermodynamic and anharmonic properties and contribute to accurate structural determination of semiconductors. The theory simplifies the many-body task into the 
one of one-dimensional model with taking the many body effects into account based on the first shell near neighbor contributions to the vibration between absorber and backscattered atoms. Morse potential contained in the derived anharmonic effective potential is assumed to describe the single-pair atomic interaction. In Section 3 the results calculated

using the present theory and those obtained by using the created method, i.e., from the available theoretical $[3,4,10]$ or experimental [5-7] second cumulants of Ge having diamond structure are compared to experiment [5-9], and to those of the other theories $[3,4,10]$, which show good agreement. The conclusions are presented in Section 4.

\section{Formalism}

\section{Anharmonic effective potential}

To determine EXAFS cumulants of a semiconductor it is necessary to specify its interatomic interaction potential and force constant. Let us consider an anharmonic interatomic effective potential expanded up to the third order around its equilibrium

$$
V_{e f f}(x)=\frac{1}{2} k_{e f f} x^{2}+k_{3 e f f} x^{3}+\ldots, x=r-r_{0}
$$

where $\mathrm{k}_{\text {eff }}$ is the effective local force constant, $\mathrm{k}_{3 \mathrm{eff}}$ is the cubic anharmonic effective parameter giving an asymmetry of the anharmonic effective potential, $\mathrm{x}$ is the deviation of the instantaneous bond length $r$ between two immediate neighboring atoms from its equilibrium value $r_{0}$.

In the present theory the many-body task is simplified into the one of one-dimensional model with taking the many-body effects into account based on including the first shell near-neighbor contributions to the vibrations between absorber and backscattered atoms. Therefore, the anharmonic effective potential Eq. (2) defined based on an assumption in the center-of-mass frame of single bond pair of an absorber and a backscattered has resulted as

$$
V_{e f f}(x) \cong \frac{7}{6} D \alpha^{2} x^{2}-\frac{35}{36} D \alpha^{3} x^{3}
$$

or in terms of parameter $\mathrm{y}=\mathrm{x}-\mathrm{a}, \mathrm{a}=\langle x\rangle$

$$
V_{e f f}(y) \cong \frac{7}{6} D \alpha^{2} y^{2}-\frac{35}{36} D \alpha^{3} y^{3}
$$

where $\alpha$ and $D$ are Morse potential parameters describing respectively the width of the potential and the dissociation energy.

Here the effective local force constant and cubic anharmonic parameter for semiconductors included in Eq. (2) as the parameters of anharmonic effective potential have been defined and given in the following forms

$$
k_{\text {eff }} \cong \frac{7}{6} D \alpha^{2}, \quad k_{3 e f f}=-\frac{35}{36} D \alpha^{3}
$$

Note that in the derivation of the above expressions we consider the diamond structure, where each atom is bonded to four nearest neighbors and Morse potential is assumed to describe the single pair atomic interaction.

For further calculation of EXAFS cumulants using quantum thermodynamic perturbation theory in the present theory the anharmonic effective potential Eq. (4) is expressed in the sum of the harmonic contribution and a perturbation $\mathrm{d} V$ due to the weak anharmonicity in EXAFS as

$$
V_{e f f}(y) \cong \frac{1}{2} k_{e f f} y^{2}+\delta V(y)
$$

where the perturbation term $\delta \mathrm{V}(\mathrm{y})$ has the following form

$$
\delta V \cong\left(k_{e f f}+3 k_{3 e f f} a^{2}\right) a y+k_{3 e f f} y^{3}
$$

\section{EXAFS cumulants}

Derivation of EXAFS cumulants in the present theory for semiconductors is based on quantum statistical theory [11] and the anharmonic effective potential derived in the previous Section 2.1. Now we consider the physical quantity which is determined based on an averaging procedure using the canonical partition function $\mathrm{Z}$ and statistical density matrix $\rho$, e.g.,

$$
\left\langle y^{m}\right\rangle=\frac{1}{Z} \operatorname{Tr}\left(\rho y^{m}\right), \quad \mathrm{m}=1,2,3, \ldots
$$

Atomic vibrations are quantized in terms of phonons, and anharmonicity is the result of phononphonon interaction, that is why we express y in terms of the annihilation and creation operators, $\hat{a}$ and $\hat{a}^{+}$, respectively

$$
\mathrm{y} \equiv \mathrm{a}_{0}\left(\hat{a}+\hat{a}^{+}\right), \quad \mathrm{a}_{0}=\sqrt{\frac{\hbar \omega_{E}}{2 k_{e f f}}}
$$

which have the following properties

$$
\left.\left[\hat{a}+\hat{a}^{+}\right]=1, \hat{a}^{+}|n\rangle=\sqrt{n+1}|n+1\rangle, \hat{a}|n=\sqrt{n-1}| n-1\right\rangle, \hat{a}^{+} \hat{a}|n\rangle=n \mid n
$$

as well as use the harmonic oscillator state $\mathrm{n}$ as the eigenstate with the eigenvalue $\mathrm{E}_{\mathrm{n}}=\mathrm{n} \hbar \omega_{E}$ for $\mathrm{n}$ being the phonon number, ignoring the zero-point energy for convenience.

Based on the local force constant given by the first equation of Eqs. (5), the correlated Einstein frequency $\omega_{E}$ and temperature $\theta_{\mathrm{E}}$ for semiconductors have resulted and given by

$$
\omega_{E}=\sqrt{\frac{2 k_{e f f}}{m}}=\sqrt{\frac{14 D \alpha^{2}}{3 m}}, \theta_{E}=\frac{\hbar \omega_{E}}{k_{B}}
$$

where $k_{B}$ is Boltzmann constant $m$ is atomic mass (for monatomic crystal). The canonical partition function in Eq. (8) can be expressed as

$$
Z \cong Z_{0}=\sum_{n} e^{-n \beta \hbar \omega_{E}}=\sum_{n=0}^{\infty} z^{n}=\frac{1}{1-z}, z=\exp \left(-\theta_{E} / T\right)
$$

Using the above results for the correlated atomic vibration and the procedure depicted by Eqs. (8) - (12), as well as the first-order thermodynamic perturbation theory [11] the 
temperature-dependent EXAFS cumulants in the present theory for semiconductors have been derived.

Here, based on the procedure depicted by Eq. (8) we derived the even moment expressing the second cumulant or MSRD

$\sigma^{2}(T)=\left\langle y^{2}\right\rangle=\sum_{n} e^{-n \beta \hbar \omega_{E}}\left\langle n\left\langle n|\delta V(y)| n^{\prime}\right\rangle n\right\rangle, \beta=1 / k_{B} T$

and the odd moments expressing the first $(\mathrm{m}=1)$ and third ( $\mathrm{m}$ =3) cumulants

$$
\left\langle y^{m}\right\rangle=\frac{k_{e f f}}{Z_{0}} \sum_{n, n^{\prime}} \frac{e^{-\beta E_{n}}-e^{-\beta E_{n^{\prime}}}}{E_{n}-E_{n^{\prime}}}\left\langle n|\delta V(y)| n^{\prime}\right\rangle\left\langle n^{\prime}\left|y^{m}\right| n\right\rangle, \quad \mathrm{m}=1,3
$$

Where $\delta V(y)$ is taken from Eq. (7) and the operations expressed by Eqs. (8) - (10) have been applied to calculate the matrix elements given in Eqs. (13) - (14).

Consequently, the EXAFS cumulants for semiconductors have resulted for the first cumulant describing net thermal expansion or lattice disorder

$$
\sigma^{(1)}(T)=a=\sigma_{0}^{(1)} \frac{1+z(T)}{1-z(T)}=\frac{\sigma_{0}^{(1)}}{\sigma_{0}^{(2)}} \sigma^{2}(T), \sigma_{0}^{(1)}=\frac{35}{83} \alpha \sigma_{0}^{2}
$$

for the second cumulant describing MSRD

$$
\sigma^{2}(T)=\left\langle y^{2}\right\rangle=\sigma_{0}^{2} \frac{1+z(T)}{1-z(T)}, \sigma_{0}^{2}=\frac{\hbar \omega_{E}}{2 k_{e f f}}
$$

and for the third cumulant describing the mean cubic relative displacement (MCRD) or the asymmetry of the pair distribution function

$$
\sigma^{(3)}(T)=\left\langle y^{3}\right\rangle=\sigma_{0}^{(3)}\left[3\left(\sigma^{2}(T) / \sigma_{0}^{2}\right)^{2}-2\right], \sigma_{0}^{(3)}=15 \alpha\left(\sigma_{0}^{2}\right)^{2}
$$

Here $\sigma_{0}^{(1)}, \sigma_{0}^{2}, \sigma_{0}^{(3)}$ are zero-point energy contributions to the cumulants $\sigma^{(1)}(\mathrm{T}), \sigma^{2}(\mathrm{~T}), \sigma^{(3)}(\mathrm{T})$, respectively.

Hence, the EXAFS quantities given by Eqs. (15) - (17) have been described in terms of second cumulant. This is useful not only for reducing the numerical calculations but also for creating a method for obtaining the remain theoretical or experimental EXAFS quantities of the considered semiconductor based on only the calculated (theoretical study) or measured (experimental study) second cumulants.

Note that the derived cumulants describe the thermodynamic and anharmonic properties and based on Fourier transform magnitudes of EXAFS spectra described by Eq. (1) containing these cumulants contribute to accurate structural determination of semiconductors.

\section{Numerical Results and Discussions}

Now we apply the expressions derived in the previous sections to numerical calculations for temperature-dependence of EXAFS cumulants of diamond structure type semiconductor Ge using its Morse potential parameters [12] D = $1.63 \mathrm{eV}, \alpha=1.50 \AA^{-1}$. The calculated values of the local force constant $\mathrm{k}_{\mathrm{eff}}$, anharmonic cubic parameter $\mathrm{k}_{3 \mathrm{eff}}$ correlated Einstein frequency $\omega_{\mathrm{E}}$ and temperature $\theta_{\mathrm{E}}$ are written in Table 1.

Table 1: Calculated values of $k_{\text {eff }}, k_{3 e f f}, \omega_{E}, \theta_{E}$ of semiconductor Ge using its Morse potential parameters [12].

\begin{tabular}{|c|c|c|c|c|}
\hline Element & $\mathbf{K}_{\text {eff }}(\mathbf{N} / \mathbf{m})$ & $\mathbf{k}_{3 \text { eff }}\left(\mathbf{e V} . \AA^{-3}\right)$ & $\omega_{E}\left(\times \mathbf{1 0}^{13} \mathbf{H z}\right)$ & $\theta_{E}(\mathbf{K})$ \\
\hline $\mathrm{Ge}$ & 137.1074 & -5.3484 & 4.7556 & 363.2636 \\
\hline
\end{tabular}

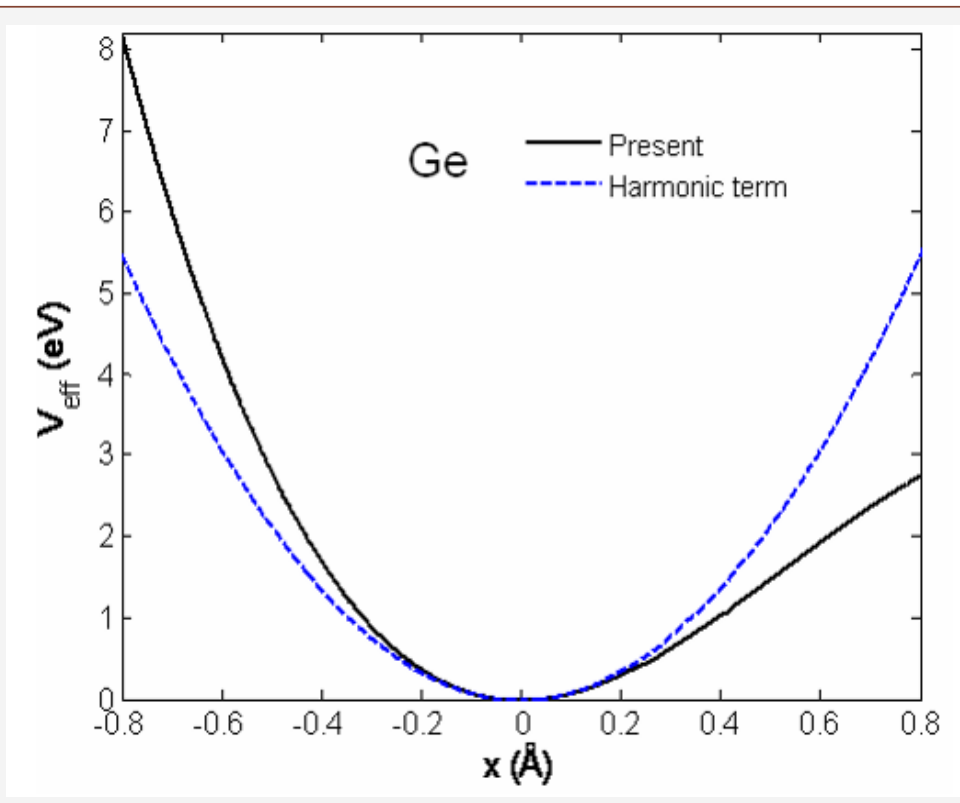

Figure 1: Anharmonic intratomic effective potentials of Ge calculated using the present theory compared to its harmonic effective term.

Figure 1 illustrates the anharmonic interatomic effective potentials of Ge calculated using the present theory. It is asymmetric due to the anharmonic contribution described by the third order parameter $\mathrm{k}_{3 \text { eff }}$ of the potential. Here, the anharmonic effective potential of Ge is compared to its harmonic effective term to show the rate of its asymmetry due to anharmonicity. This anharmonic effective potential of $\mathrm{Ge}$ is used for calculating the considered EXAFS quantities.

Temperature-dependence of second cumulant or MSRD $\sigma^{2}(\mathrm{~T})$ of Ge (Figure 2) calculated using the present theory agrees well 
with the experimental values of G Dalba, et al. (Expt.) [5,6], and of AE Stern, et al. [7], with the result of FD Vila, et al. [3] and of JJ Rehr, et al. [4] calculated using the LDA (local density approximation), and of NV Hung, et al. [10] calculated using the SMM (statistical moment method) and Stillinger-Weber potentials.

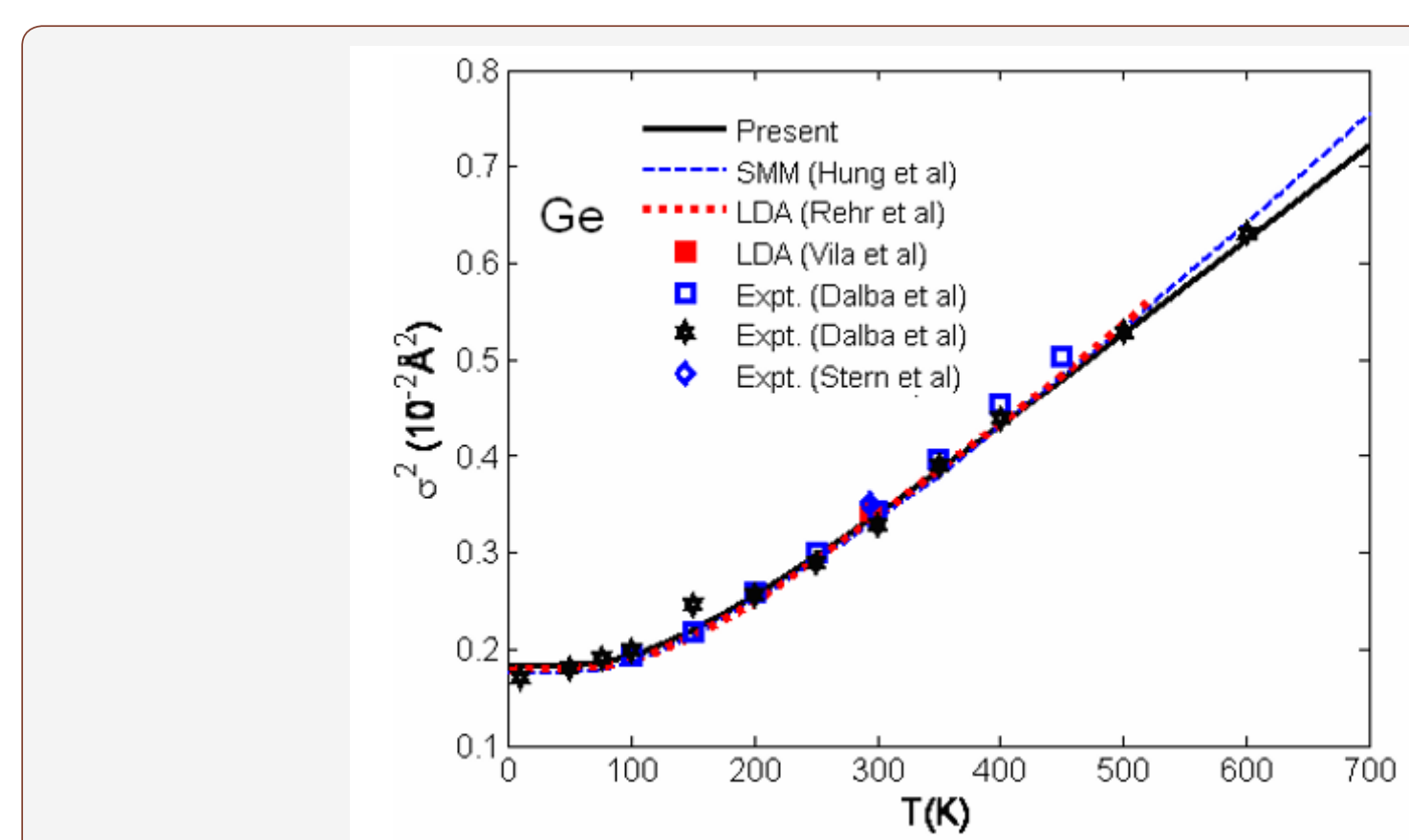

Figure 2: Temperature-dependence of second cumulant or MSRD $\sigma^{2}(T)$ calculated using the present theory for Ge compared to the experimental EXAFS values of G Dalba, et al. (Expt.) [5,6] and of EA Stern, et al. [7], as well as to those of FD Vila, et al. [3] and of JJ Rehr, et al. [4] calculated using LDA, and of NV Hung, et al. [10] calculated using SMM and Stillinger-Weber potential.

Temperature-dependence of the first cumulant or net thermal expansion $\sigma^{(1)}(\mathrm{T})$ of Ge (Figure 3) calculated using the present theory agrees well with the experimental values of G Dalba, et al. [8], and to those calculated from the experimental (exp.) MSRD of G Dalba, et al. [5], of EA Stern, et al. [7], as well as from the calculated (calc.) MSRD of FD Vila, et al. [3], and of JJ Rehr, et al. [4] calculated using the LDA. Such good agreement is also shown for the third cumulant $\sigma^{(3)}(\mathrm{T})$ of Ge (Figure 4) calculated using the present theory with the EXAFS experimental values of P Fornasini, et al. [9], as well as to those calculated from the experimental (exp.) MSRD of Dalba, et al. [6], of E Stern, et al. [7] and from the calculated (calc.) MSRD of FD Vila, et al. [3], and of JJ Rehr, et al. [4] calculated using the LDA.

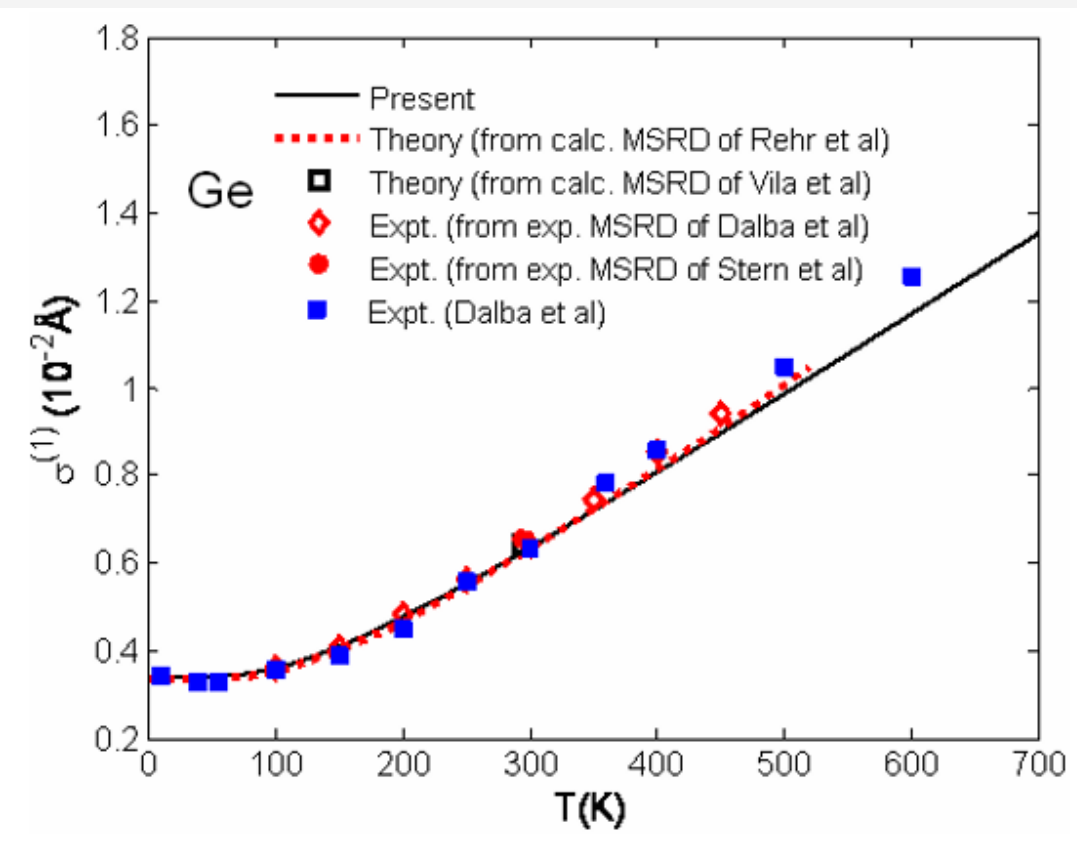

Figure 3: Temperature-dependence of first cumulant ${ }^{\sigma(1)}(T)$ calculated using the present theory for Ge compared to the experimental values of G Dalba, et al. [8], and to those calculated from the experimental (exp.) MSRD of G Dalba, et al .[5], of EA Stern, et al. [7], as well as from the calculated (calc.) MSRD of FD Vila, et al. [3], and of JJ Rehr, et al. [4] calculated using the LDA. 
Note that the good agreement of the values calculated from the theoretical and experimental second cumulants or MSRDs of Ge (Figure 2) for the first cumulants presented in Figure 3 and for the third cumulants presented in Figure 4 with experiment confirms not only their validity as the theoretical and experimental EXAFS quantities but also the possibility of getting the other EXAFS parameters if the second cumulants are calculated or measured. This actually also confirms the validity of the above created method which has been used in the present studies.

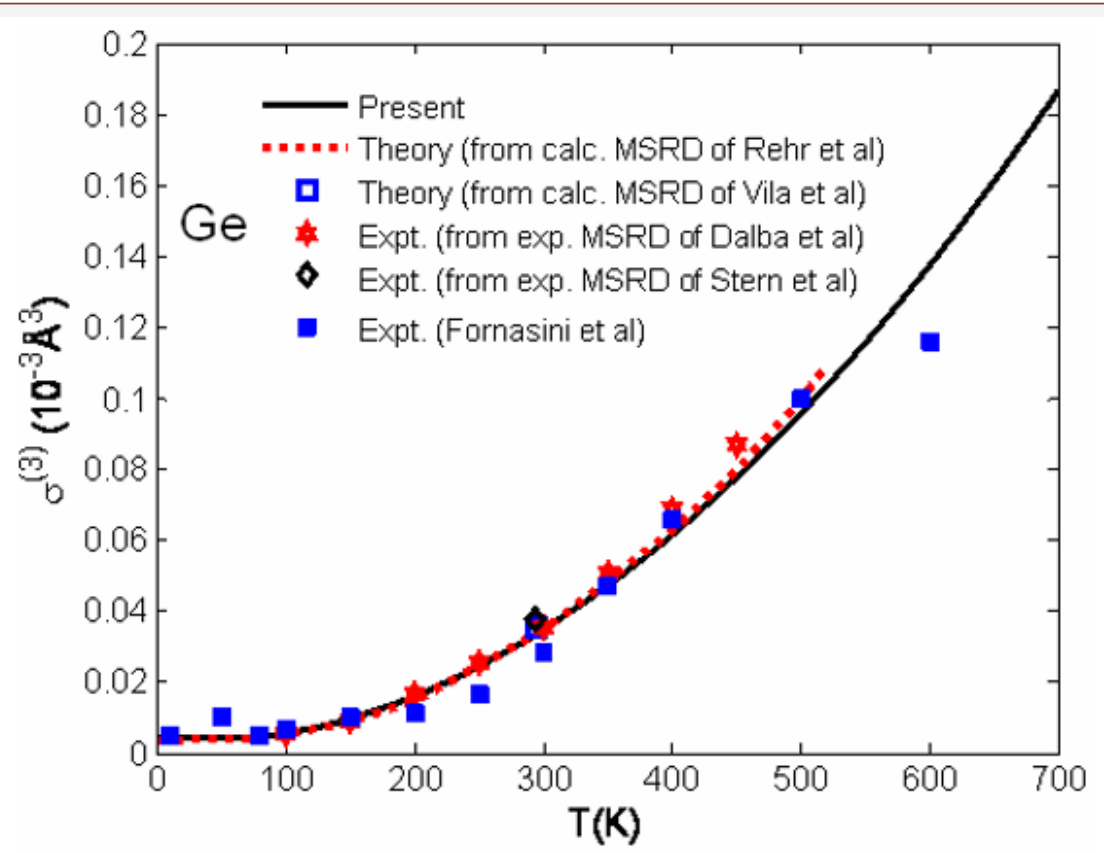

Figure 4: Temperature-dependence of third cumulant $\sigma^{(3)}(T)$ calculated using the present theory for Ge compared to the EXAFS experimental values of Fornatsini, et al. [9], as well as to those calculated from the experimental (exp.) MSRD of Dalba et al [6], of E Stern, et al. [7] and from the calculated (calc.) MSRD of FD Vila, et al. [3], and of JJ Rehr. et al [4] calculated using the LDA.

The above figures show that at high temperatures the first and second cumulants are linearly proportional to temperature $\mathrm{T}$ and the third cumulant to $\mathrm{T}^{2}$. They contain zero-point energy contributions at low-temperatures, a quantum effect.

The cumulant ratios are often considered in the anharmonic EXAFS studies $[2,13,14]$. Figure 5 illustrates temperature- dependence of cumulant ratio $\sigma^{(1)} \sigma^{2} / \sigma^{(3)}$ calculated using the present theory for Ge. Here, $\sigma^{(1)} \sigma^{2} / \sigma^{(3)}$ approaches $3 / 2$ at low-temperatures and the classical value $[13,14]$ of $1 / 2$ athigh temperatures beginning from the Einstein temperature $\left(\theta_{\mathrm{E}}=363.26 \mathrm{~K}\right.$ for Ge, Table 1$)$ as for the other crystal structures [2]. Hence, it remains to be considered as the standard for studying EXAFS cumulants of semiconductors calculated using the present theory.

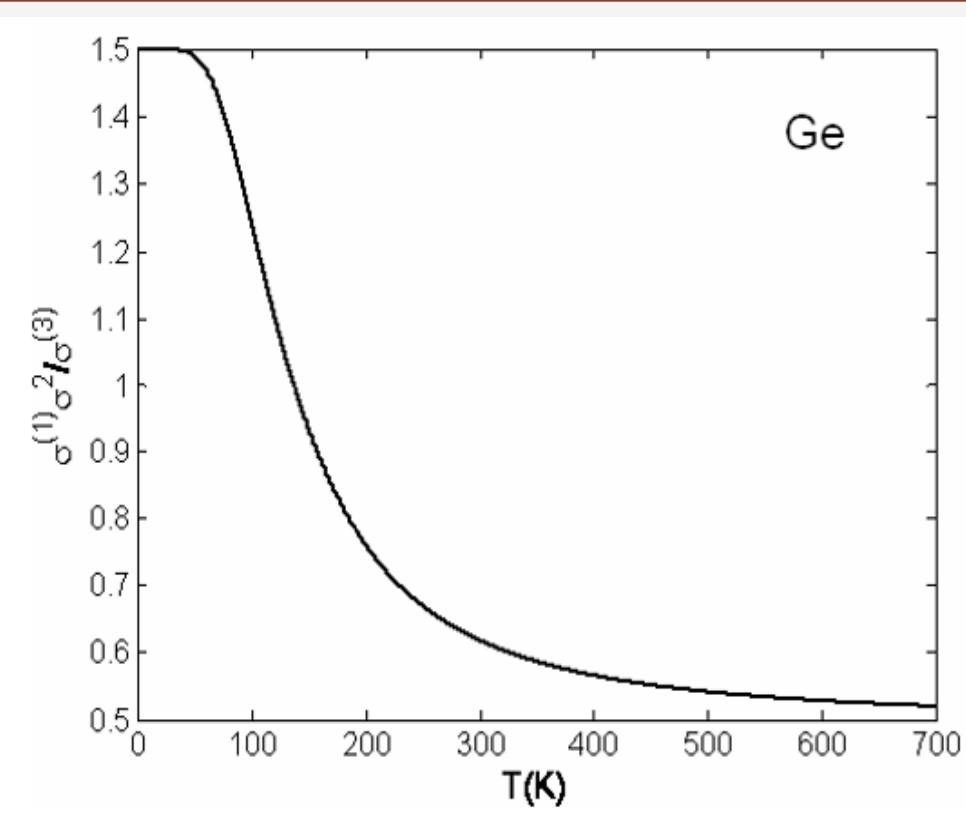

Figure 5: Temperature-dependence of cumulant ratio $\sigma^{(1)} \sigma^{2} / \sigma^{(3)}$ of Ge calculated using the present theory. 


\section{Conclusion}

In this work a theory has been derived for studies of temperature-dependence of DWFs of semiconductors presented in terms of cumulant expansion in EXAFS. They contribute to accurate structural determination, as well as to valuation of the thermodynamic properties and anharmonic effects accounting for the net thermal expansion, the MSRD, and the asymmetry of the pair distribution function of semiconductors.

The advanced studies are succeeded based on the further development of the ACEM derived primary for fcc crystals into the one applied to semiconductors. Here the many-body task is simplified into the one of one-dimensional model with taking the many-body effects into account based on including the first shell near-neighbor contributions to the vibrations between absorber and backscattered atoms.

The method created in this theory has the advantage that all theoretical and experimental EXAFS quantities are provided based on only the calculation (theoretical study) or measurement (experimental study) of second cumulants. It is useful not only for reducing the numerical calculations or measurements but also for creating a simple way of obtaining the considered quantities.

The theory is successfully applied to valuation of temperaturedependence of three first EXAFS cumulants of semiconductors. At high temperatures the first and second cumulants are linearly proportional to temperature $\mathrm{T}$ and the third one to $\mathrm{T}^{2}$. They contain zero-point energy contributions at low-temperatures, a quantum effect. The cumulant ratio $\sigma^{(1)} \sigma^{2} / \sigma^{(3)}$ expresses the same properties as those for the other crystal structures so that it remains to be considered as the standard for EXAFS cumulant studies of semiconductors.

The good agreement of the results calculated using the present theory and of those obtained by using the created method with experiment and with those of other theories for Ge having diamond structure illustrates the simplicity, advantage and efficiency of the present theory and the created method in the theoretical and experimental EXAFS studies of the thermodynamic properties and anharmonic effects of materials having any structure including semiconductors.

\section{Acknowledgement}

The authors thank JJ Rehr \& P Fornasini for useful comments and appreciate $\mathrm{P}$ Fornasini for providing experimental data of EXAFS cumulants of Ge. This research is funded by the VNU University of Science under project number TN.19.21.

\section{Conflict of interest}

On behalf of all authors the corresponding author states there is no conflict of interest.

\section{References}

1. ED Crozier, JJ Rehr, R Ingalls (1988) in X-ray Absorption, edited by DC Koningsberger, R Prins, Wiley, New York, Chap 9.

2. NV Hung, JJ Rehr (1997) Anharmonic correlated Einstein-model DebyeWaller factors. Phys Rev B 56: 43.

3. FD Vila, JJ Rehr, HH Rossner, HJ Krappe (2007) Theoretical x-ray absorption Debye-Waller factors. Phys Rev B 76: 014301.

4. JJ Rehr, JJ Kas, MP Prange, AP Sorini, Y Takimoto, FD Villa (2009) CR Physique 10: 548.

5. G Dalba, P Fornasini, D Diop, M Grazioli, F Rocca (1993) J Non-Crys Sol 164: 159 .

6. G Dalba, P Fornasini, R Grisenti, J Purans (1999) Sensitivity of Extended X-Ray-Absorption Fine Structure to Thermal Expansion. Phys Rev Lett 82: 4240 .

7. AE Stern, BA Bunker, SM Heald (1980) Many-body effects on extended X-ray absorption fine structure amplitudes. Phys Rev B 21: 5521.

8. G Dalba, P Fornasini, R Grisenti, J Purans (1999) J Synch Rad 6: 532.

9. P Fornasini, G Dalba, R Grisenti, J Purans J, A Sanson, et al. (2004) Phys Stat Sol (c) 1: 3085.

10. NV Hung, CS Thang, NC Toan, HK Hieu (2014) VAC 101: 63.

11. RF Feynman (1972) Statistical Mechanics, edited by Jacob Shaham, WA Benjamin, INC Advanced book Program, Reading, Massachusetts.

12. RA Swalin (1961) J Phys Chem Solids 18: 290.

13. EA Stern, P Livins, Zhe Zhang (1991) Phys Rev B 43: 8850.

14. NV Hung, TS Tien, NB Duc, DQ Vuong (2014) Mod Phys Lett B 28: 1450174 